\title{
Thermal assessment of sunlight impinging on OSIRIS-REx OCAMS PolyCam, OTES, and IMU-sunshade MLI blankets in flight
}

\author{
Michael K. Choi*a
}

${ }^{a}$ NASA Goddard Space Flight Center, Greenbelt, MD 20771, USA

\begin{abstract}
The NASA Origins, Spectral Interpretation, Resource Identification, Security, Regolith Explorer (OSIRIS-REx) spacecraft was successfully launched into orbit on September 8, 2016. It is traveling to a near-Earth asteroid (101955) Bennu, study it in detail, and bring back a pristine sample to Earth for scientific analyses. At the Outbound Cruise nominal spacecraft attitude, with Sun on $+X$, sunlight impinges on the OSIRIS-REx camera suite (OCAMS) PolyCam sunshade multilayer insulation (MLI) with microporous black polytetrafluoroethylene (PTFE), a portion of the PolyCam optics support tube (MLI with germanium black Kapton (GBK)), a portion of the OSIRIS-REx Thermal Emission Spectrometer (OTES) sunshade (MLI with GBK), the Inertia Measurement Unit (IMU) sunshade (MLI with GBK), and the OSIRIS-REx Laser Altimeter (OLA) sunshade (MLI with GBK). Sunlight is reflected or scattered by the above MLIs to the other components on the forward $(+Z)$ deck. It illuminates the forward deck. A detailed thermal assessment on the solar impingement has been performed for the Proximity Ops at the asteroid, Touch-and-Go (TAG) sample acquisition, and Return Cruise mission phases.
\end{abstract}

Keywords: solar impingement on MLI, stray light thermal effect, microporous black PTFE, diffuse MLI, specular MLI, sunlight reflected by MLI, asteroid sample return mission, OSIRIS-REx

\section{INTRODUCTION}

The NASA OSIRIS-REx spacecraft was successfully launched into orbit on September 8, 2016. It is traveling to a nearEarth asteroid (101955) Bennu, study it in detail, and bring back a pristine sample of larger than $60 \mathrm{~g}$ and up to $2 \mathrm{~kg}$ to Earth for scientific analyses. Figure 1 Presents the Mission Phases, including the Sun range, which affects the spacecraft's thermal environment. The duration of the Outbound Cruise is 712 days.

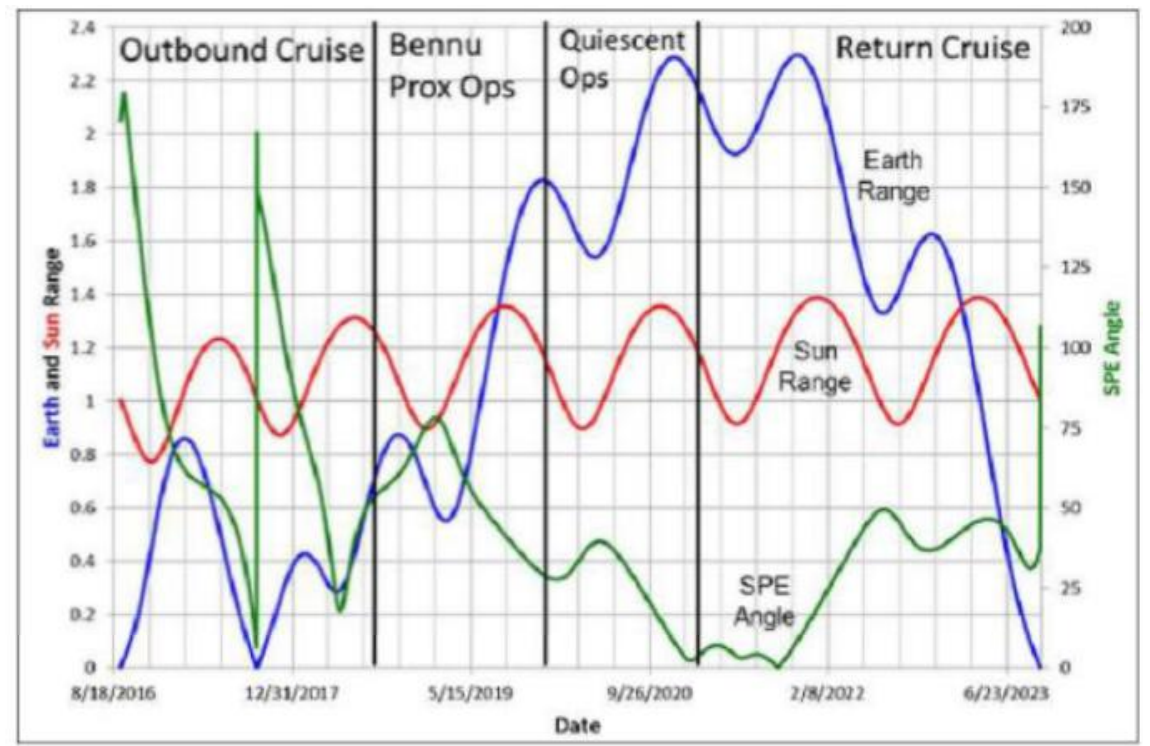

Figure 1. OSIRIS-REx Mission Phases (source: NASA, OSIRIS-REx Project).

*michael.k.choi@nasa.gov; phone 1301 286-4707; fax 1 301 286-1704 
At the Outbound Cruise nominal spacecraft Sun-pointing attitude (i.e., Sun on $+X$ ), sunlight is incident on the following components on the forward deck:

- The OCAMS PolyCam sunshade MLI with microporous black PTFE.

- A portion of the PolyCam optics support tube (MLI with GBK).

- A portion of the OTES sunshade (MLI with GBK).

- The spacecraft IMU-sunshade (MLI with GBK).

- The spacecraft OLA-sunshade (MLI with GBK).

Figures 2 through 4 illustrate the solar impingement.

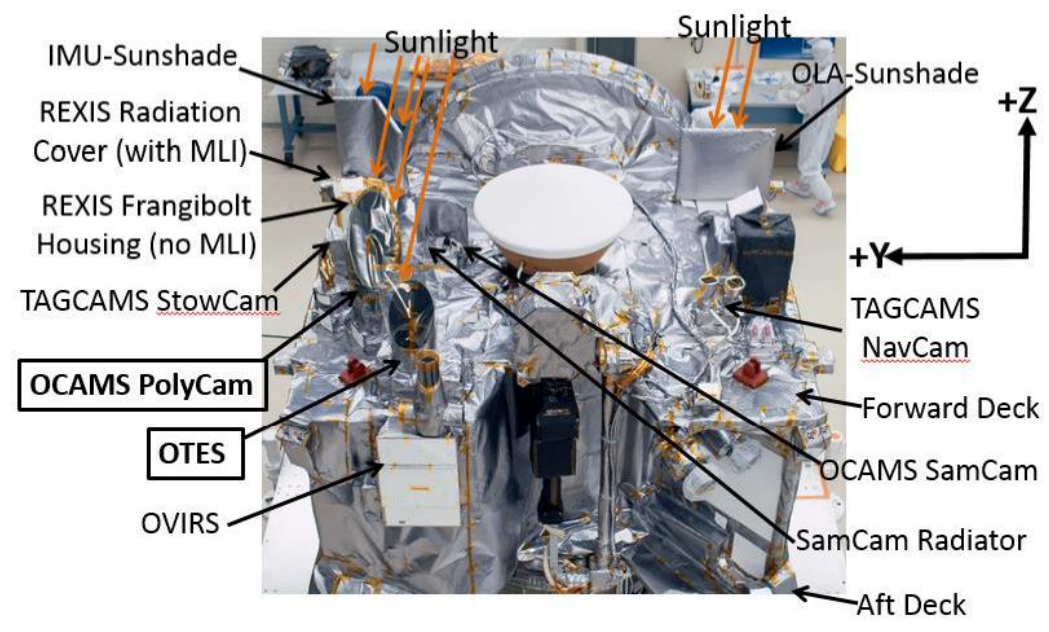

Figure 2. Solar Impingement on OCAMS PolyCam and OTES (View from $-\mathrm{X} /+\mathrm{Z}$ ).

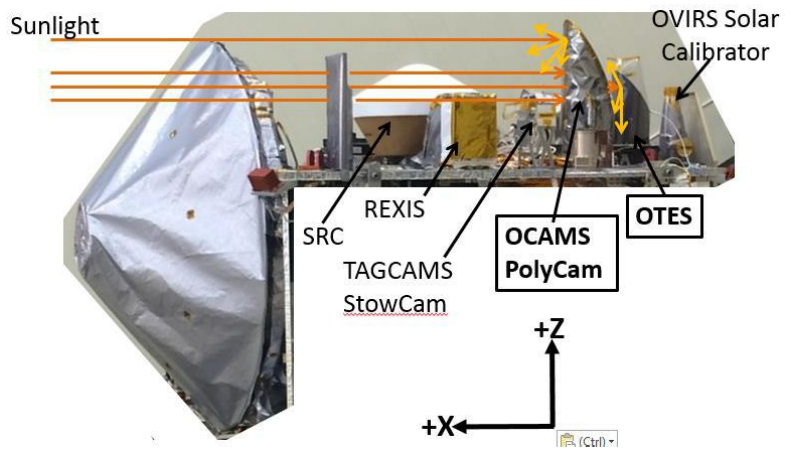

Figure 3. Solar Impingement on OCAMS PolyCam and OTES (View from + Y).

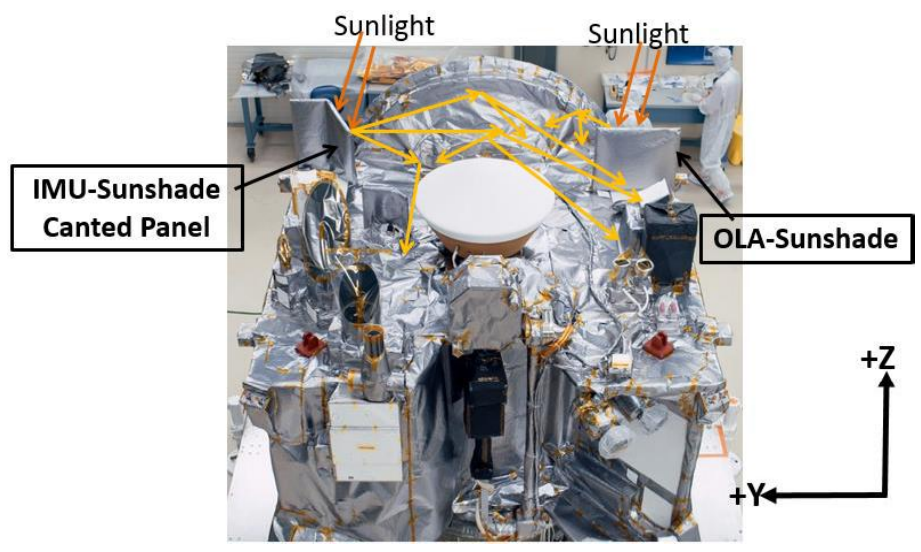

Figure 4. Solar Impingement on IMU-Sunshade and OLA-Sunshade (View from -X/+Z). 
Microporous black PTFE ${ }^{1}$ is used as a cover for the PolyCam sunshade MLI. It is to mitigate the issue of reflecting stray light into the MapCam during the dust-plume survey because stray light would look the same as a dust plume. The microporous black PTFE sheet used on OSIRIS-REx has a solar absorptance of 0.97 and a hemispherical emittance of 0.86 . It reflects only $3 \%$ of the sunlight incident on it. Additionally, it is optically diffuse (Figure 5). The GBK used on OSIRIS-REx has a solar absorptance of 0.51 and a hemispherical emittance of 0.84 . It reflects $49 \%$ of the sunlight incident on it. Additionally, it is optically very specular. Its specularity was measured to be 69\%. Figure 6 illustrates the optical difference between microporous black PTFE and GBK when light is incident on them. Sunlight, which impinges on the PolyCam, OTES, IMU-sunshade and OLA-sunshade MLIs, is reflected or scattered by the GBK for the most part, and microporous black PTFE to the other components on the forward deck. It illuminates the forward deck. The OCAMS cameras experienced scattered light in the September 19, 2016 spacecraft configuration. ${ }^{2}$ It was successfully removed from images by median filter.

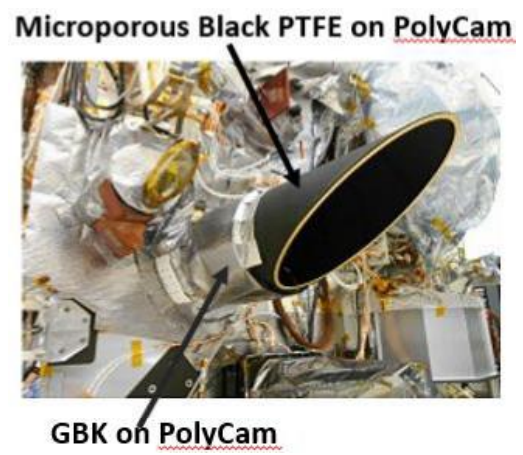

Figure 5. Microporous black PTFE and GBK on PolyCam.

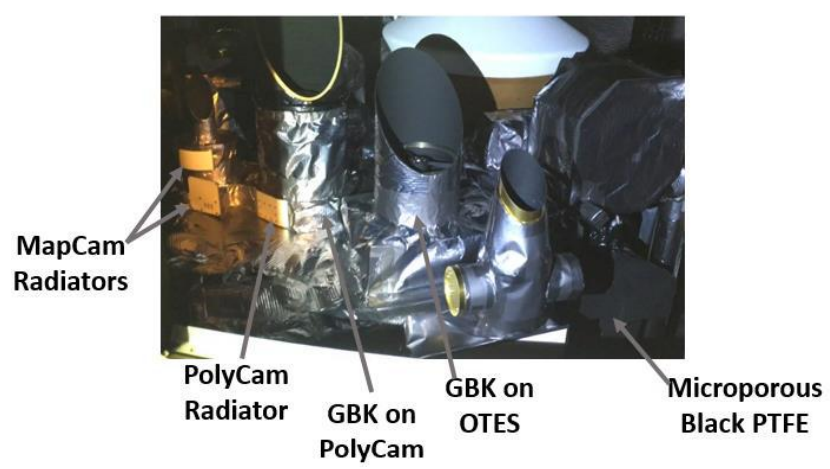

Figure 6. Optical Difference between Microporous black PTFE and GBK.

Figure 7 shows an image taken by the TAG Camera System (TAGCAMS) StowCam camera two weeks after launch. Stray light, which illuminates the Sample Return Capsule (SRC) and nearby components, is seen in the image. If GBK were used for the PolyCam sunshade MLI, the stray light would be much brighter because GBK has a much lower solar absorptivity and is highly specular.

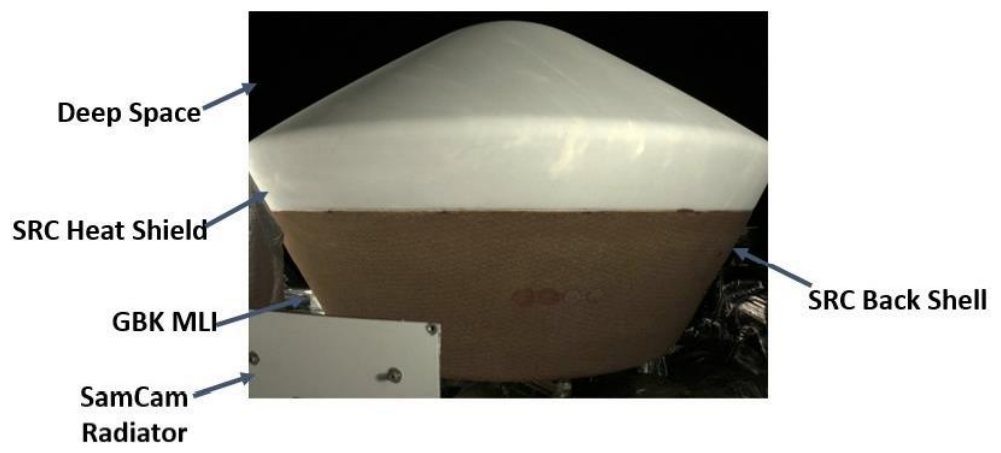

Figure 7. Image Taken by TAGCAMS StowCam (source: OSIRIS-REx Project). 


\section{OBJECTIVES}

The objectives of this paper are:

-To present a detailed thermal assessment on the above solar impingement, and

-To present the thermal implications of the above solar impingement for the OSIRIS-REx mission phases after the Outbound Cruise: Proximity Ops at asteroid Bennu, TAG sample acquisition, and Return Cruise.

\section{THERMAL IMPACT OF SOLAR IMPINGEMENT IN OUTBOUND CRUISE}

At the OSIRIS-REx Outbound Cruise nominal spacecraft attitude, sunlight impinges on the OCAMS PolyCam sunshade MLI, a portion of the PolyCam optics support tube MLI, a portion of the OTES sunshade MLI, the IMU-sunshade MLI, and the OLA-sunshade MLI. It causes warming of these MLIs, which radiates heat (infrared) to the other components within their views. Additionally, the solar impingement is reflected or scattered by the above MLIs to the other components on the forward deck as well as the forward deck. The intensity of the solar impingement is strongly dependent on the solar distance. In the Outbound Cruise, which is nearly two years long, the solar distance is in the 0.773 astronomical unit (AU) and 1.387 AU range. Figure 8 shows the solar distance versus the number of days after launch. Solar irradiance is inversely proportional to the square of the solar distance. Figure 9 shows the solar irradiance versus the number of days after launch.

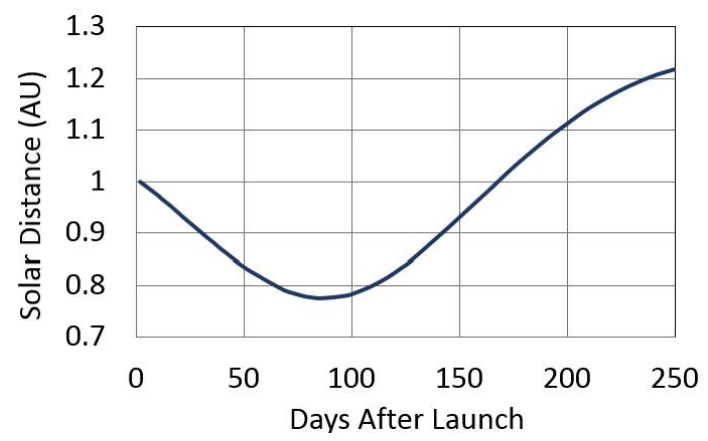

Figure 8. Solar Distance versus Days after Launch.

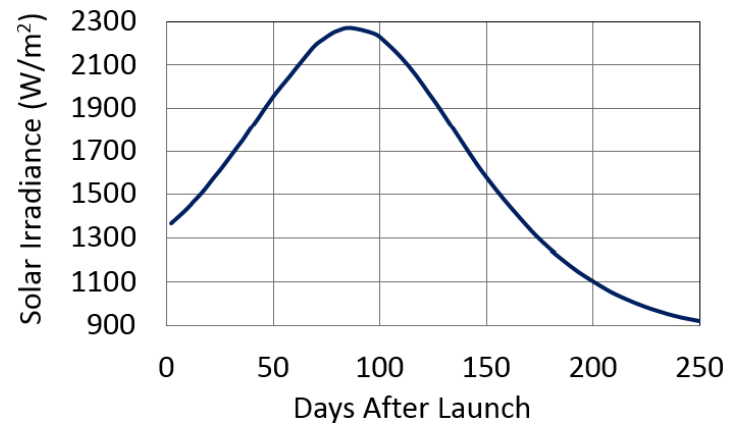

Figure 9. Solar Irradiance versus Days after Launch.

The thermal impact of the solar impingement on selected components is discussed below.

\subsection{Forward (+Z) Deck}

The OSIRIS-REx forward deck accommodates the instruments, TAG Sample Acquisition Mechanism (TAGSAM)/SRC assembly, and spacecraft guidance, Navigation and Control (GN\&C) components. Except for radiators, the forward deck is covered with GBK MLI blankets. Like the forward deck, the aft (-Z) deck is covered with GBK MLI blankets. Figure 10 shows a plot of Sun on the X, Y and Z axes from Day 030 to Day 036 in 2017. Figure 11 shows the temperatures of the forward deck and aft deck from Day 030 to Day 036 in 2017. When Sun was at a $20^{\circ}$ angle with $-Z$ during the offnominal spacecraft Earth-pointing attitude, the forward deck temperature decreased and the aft deck temperature increased. After the spacecraft attitude returned to the nominal Outbound Cruise sun-pointing (i.e., Sun on $+\mathrm{X}$ ), the 
forward deck temperature increased and the aft deck temperature decreased. As a result, the temperatures of the forward deck and aft decks oscillated. Additionally, during the nominal Outbound Cruise attitude with Sun on $+X$, the forward deck temperature is dependent on the solar distance. As the solar distance decreased, the forward deck temperature increased, and vice versa. Figure 12 shows this trending. It is evident that with Sun on $+X$, sunlight impinging on the PolyCam, OTES, IMU-sunshade and OLA-sunshade MLIs is reflected or scattered to the GBK MLI on the forward deck. As a result, it increases the MLI temperature, which in turns increases the forward deck temperature.

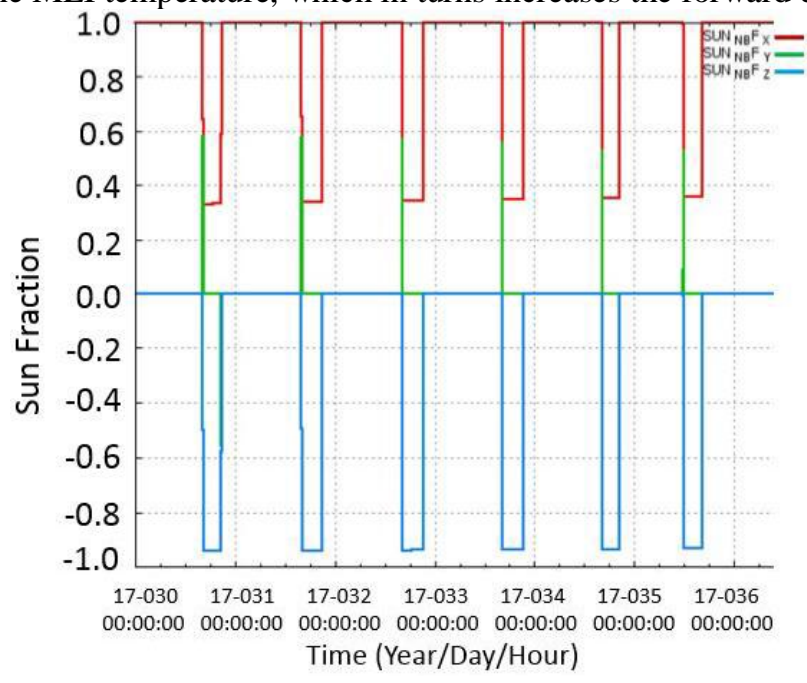

Figure 10. Fraction of Sun on X, Y and Z Axes from Day 030 to Day 036 in 2017.

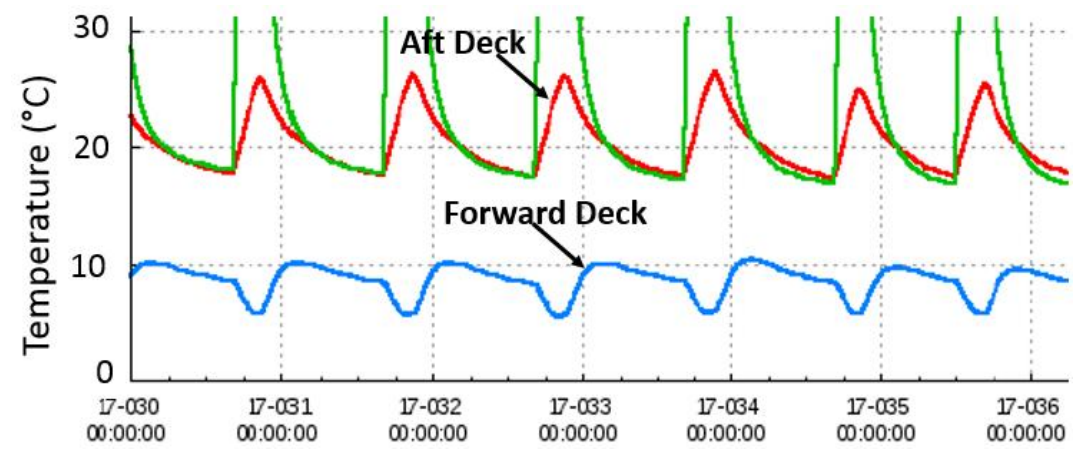

Figure 11. Forward Deck and Aft Deck Temperatures from Day 030 to Day 036 in 2017.

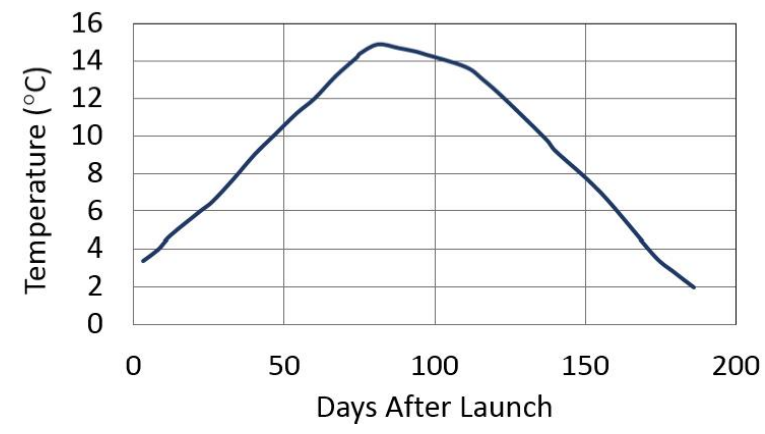

Figure 12. Forward Deck Temperature versus Days after Launch.

\subsection{TAG Camera System (TAGCAMS)}

The TAGCAMS is an OSIRIS-REx spacecraft GN\&C component. It includes three wide field cameras: two NavCam units and a StowCam unit. The NavCam units are located at the -Y side of the forward deck. The StowCam is located at the $+Y$ side of the forward deck. It is close to the OCAMS PolyCam and not very far from the OTES. The locations can 
be seen in Figure 2. At the nominal Outbound Cruise Sun-pointing attitude (i.e., Sun on $+X$ ), both the OCAMS PolyCam and OTES MLIs receive solar impingement and reflect or scatter it to the other components on the forward deck. Additionally, as these two MLIs are heated by the sun, they radiate heat (infrared) to the components within their views. Therefore, the thermal environment for the StowCam is warmer than that for the NavCam. The difference in radiator sizes also contributes to a thermal difference. The non-operating (survival) heaters on the TAGCAMS cameras are controlled by the spacecraft flight software. The heater controller set point is $-38^{\circ} \mathrm{C}$ on and $-36^{\circ} \mathrm{C}$ off. Each camera has its own temperature sensor and heater, but does not have its own heater controller. The spacecraft flight software compares the temperatures of the three temperature sensors, one on each camera, and uses the coldest one to control the heaters. During the nominal Outbound Cruise Sun-pointing attitude, flight temperature telemetry shows there is a large temperature difference between the NavCam and StowCam (Figure 13, which corresponds to Figure 8). The difference between maximum and minimum is due to heater cycling. The StowCam temperature increases as the solar distance decreases, and vice versa, except from 87 to 93 days after launch. From Figure 13, with the TAGCAMS non-operating, the temperatures of the NavCam cameras were maintained between $-38.2^{\circ} \mathrm{C}$ and $-35^{\circ} \mathrm{C}$ by survival heaters, except from 87 to 93 days after launch. The survival heaters stopped drawing current from 87 to 93 days after launch because the NavCam temperature was warmer than the $-38^{\circ} \mathrm{C}$ heater controller set point. As a result, the StowCam and NavCam temperatures were not affected by heater duty cycling during those six hours. When the NavCam survival heaters were on, the StowCam survival heater was also on, despite its temperature was much warmer than $-38^{\circ} \mathrm{C}$ and did not require heater power. Figure 14 shows the TAGCAMS temperatures from Day 030 to Day 036 in 2017. It corresponds to Sun on $\mathrm{X}, \mathrm{Y}$ and $\mathrm{Z}$ in Figure 10. From Figure 14, when the spacecraft attitude changed from Sun-pointing to Earth-pointing, the StowCam temperature decreased, and vice versa. As a result, the StowCam temperatures oscillated. Changes of the spacecraft attitude from Sun-pointing to Earth-pointing increase the NavCam heater duty cycle, and vice versa. It is evident that solar impingement on the PolyCam, OTES, IMU-sunshade and OLA-sunshade MLIs affects the StowCam temperature and NavCam heater duty cycle.

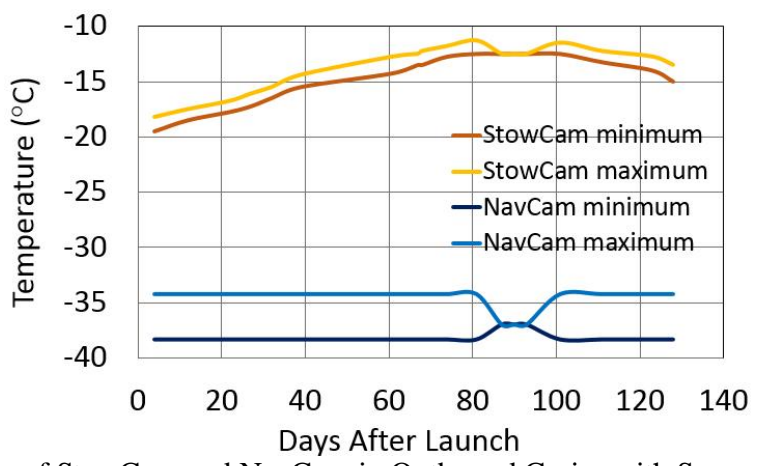

Figure 13. Flight Temperature of StowCam and NavCam in Outbound Cruise with Sun on $+X$.

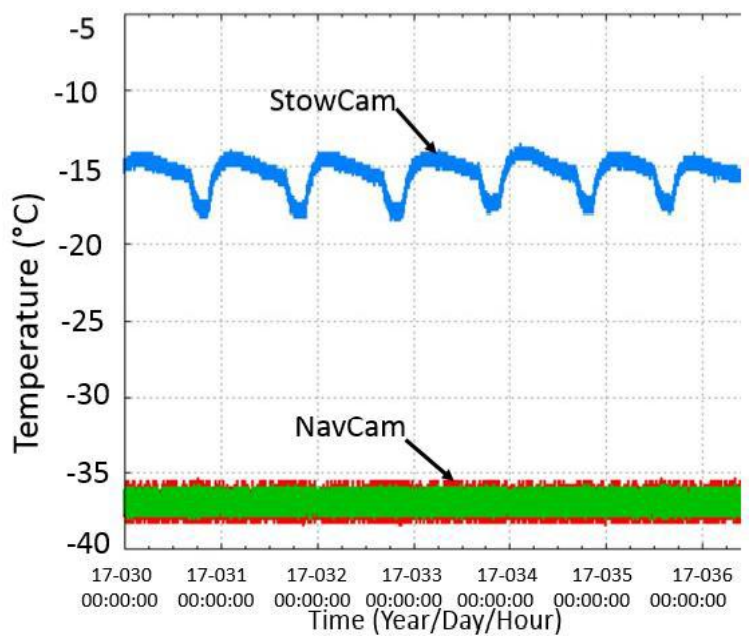

Figure 14. StowCam and NavCam Temperatures from Day 030 to Day 036 in 2017. 


\subsection{OSIRIS-REx Camera Suite (OCAMS)}

\subsubsection{Cameras}

The OCAMS is the primary instrument of the OSIRIS-REx mission. It is located at the $+\mathrm{Y}$ side of the forward deck (Figure 2). It consists of three high-resolution cameras (MapCam, PolyCam and SamCam) and a camera control module (CCM). The cameras are thermally isolated from the baseplates on the spacecraft side by titanium flexures. A radiator, which has Z93C55 white paint as the thermal coating, is mounted to each camera chassis. It cools the charged couple device (CCD) and electronics passively. The survival heaters are located on the instrument side and controlled by the spacecraft flight software. All the OCAMS survival heater resistances were sized for low duty cycles (30\% or less). Figure 15, Figure 16 and Figure 17 show the CCD temperatures of the MapCam, PolyCam and SamCam, respectively, from Day 030 to Day 036 in 2017. They corresponds to Sun on X, Y and Z in Figure 10. Figure 16 also shows the temperatures of the PolyCam Optics. The MapCam CCD temperature was maintained between $-26^{\circ} \mathrm{C}$ and $-21.5^{\circ} \mathrm{C}$ by its survival heaters. The PolyCam CCD temperature was maintained between $-25.5^{\circ} \mathrm{C}$ and $-18.3^{\circ} \mathrm{C}$ by its survival heaters. The PolyCam Optics temperature was maintained between $-36.5^{\circ} \mathrm{C}$ and $-31.4^{\circ} \mathrm{C}$ by its survival heaters. The SamCam CCD temperature was maintained between $-21.3^{\circ} \mathrm{C}$ and $-15.5^{\circ} \mathrm{C}$ by its survival heaters. Changes of the spacecraft attitude from Sun-pointing to Earth-pointing, or vice versa, affected the duty cycle of the survival heaters on the CCD of each camera. The low heater resistances causes high peak heater power and low duty cycle. As a result, it affects the timing of the flight software heater control. During the nominal Sun-pointing attitude, the heater duty cycle decreased such that the CCD temperature band, which is the difference between the maximum and minimum, became smaller. During the off-nominal Earth-pointing attitude, the heater duty cycle increased such that the CCD temperature band became larger, and the PolyCam Optics temperature band shift about $1{ }^{\circ} \mathrm{C}$ colder. As a result, the temperatures of the camera CCDs and PolyCam Optics oscillated. It is evident that solar impingement on PolyCam, OTES, IMU-sunshade and OLA-sunshade MLIs has a thermal impact on the OCAMS cameras.

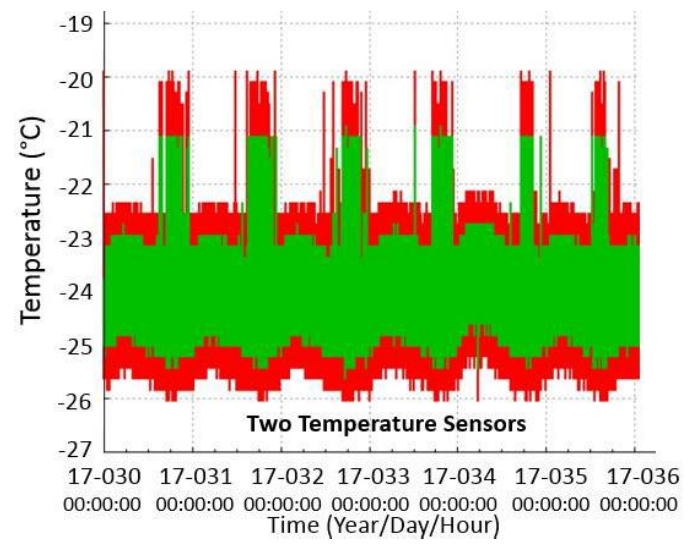

Figure 15. MapCam CCD Temperature from Day 030 to Day 036 in 2017.

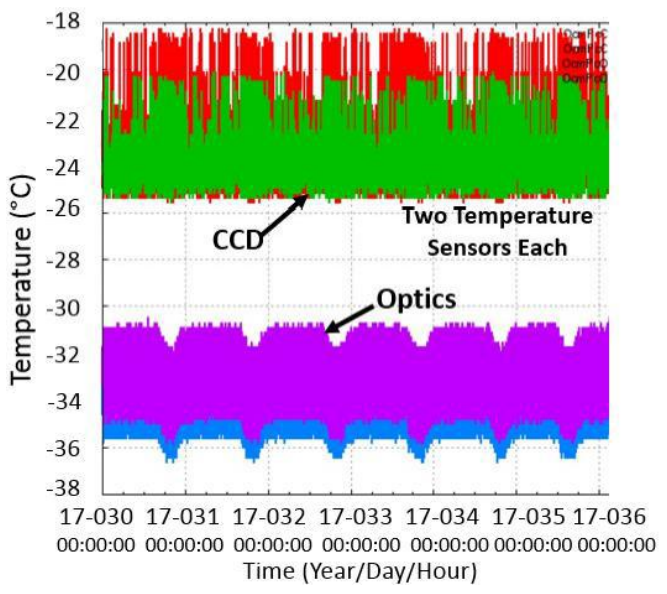

Figure 16. PolyCam CCD and Optics Temperatures from Day 030 to Day 036 in 2017. 


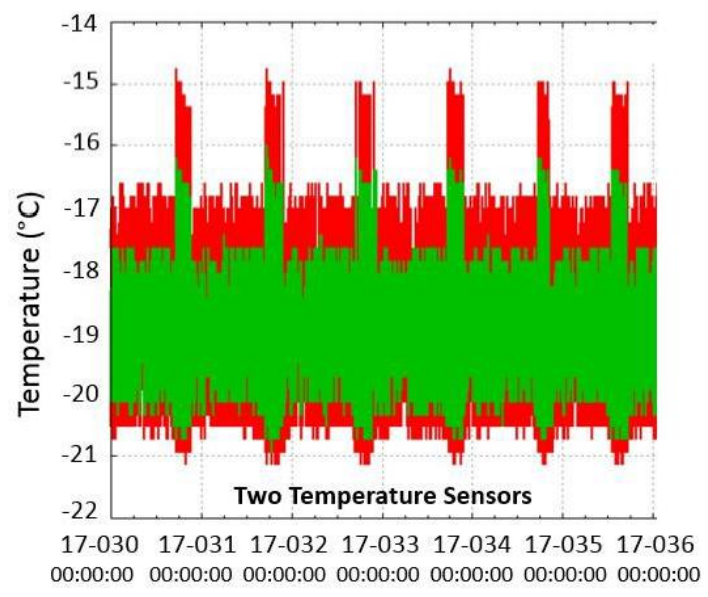

Figure 17. SamCam CCD Temperature from Day 030 to Day 036 in 2017.

\subsubsection{Camera Baseplates}

Figure 18 shows the temperatures of the PolyCam and SamCam baseplates from Day 030 to Day 036 in 2017. When the spacecraft attitude changed from Sun-pointing to Earth-pointing, the PolyCam and SamCam baseplate temperatures decreased, and vice versa. As a result, the PolyCam and SamCam baseplate temperatures oscillated. Additionally, during the nominal Outbound Cruise Sun-pointing attitude, the PolyCam and SamCam baseplate temperatures are dependent on the solar distance. As the solar distance decreases, the PolyCam and SamCam baseplate temperatures increase, and vice versa. Figure 19 shows this trending. It is evident that with Sun on $+\mathrm{X}$, solar impingement on the PolyCam, OTES and IMU-sunshade and OLA-sunshade MLIs is reflected or scattered to the GBK MLI over the PolyCam and SamCam baseplates. As a result, it increases the MLI temperature, which in turns increases the PolyCam and SamCam baseplate temperatures.

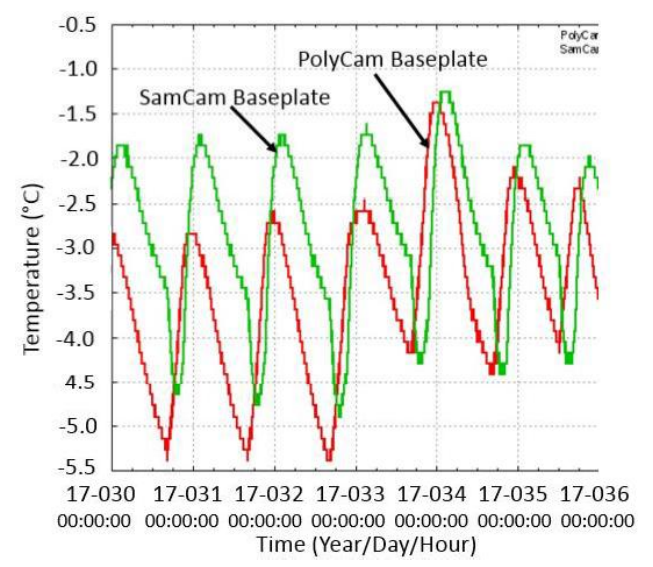

Figure 18. PolyCam Baseplate and SamCam Baseplate Temperatures from Day 030 to Day 036 in 2017.

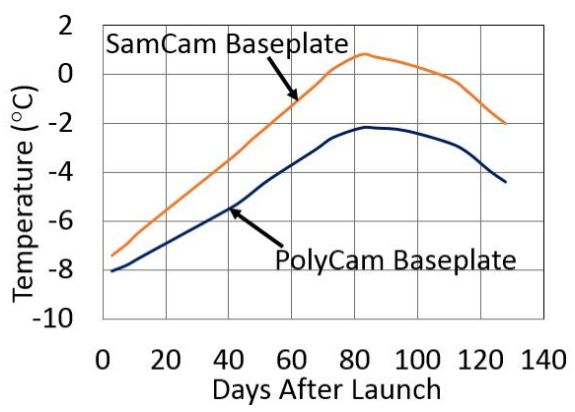

Figure 19. PolyCam Baseplate and SamCam Baseplate Temperatures from Day 030 to Day 036 in 2017. 


\subsection{Sample Return Capsule (SRC)}

The OSIRIS-REx SRC consists of five components: heat shield, back shell, sample canister, parachute system, and avionics. The sample canister is inside the back shell. The parachute and Sep Nut are outside the back shell. Figure 20 shows the temperatures of the Sep Nut, Parachute and Canister versus days after launch. The relationship between solar distance and days after launch can be seen in Figure 8. As the solar distance decreases, the temperatures of the Parachute, Sep Nuts and Canister increase. Conversely, as the solar distance increases, the temperatures of Parachute, Sep Nuts and Canister decrease. Figure 21, Figure 22 and Figure 23 show the temperatures of the Sep Nut, Parachute and Canister, respectively, from Day 030 to Day 036 in 2017. They corresponds to Sun on X, Y and Z in Figure 10. When the spacecraft attitude changed from Sun-pointing to Earth-pointing, the temperatures of these SRC components decreased, and vice versa. As a result, the temperatures of these SRC components oscillated. It is evident that sunlight impinging on the PolyCam, OTES, IMU-sunshade and OLA-sunshade MLIs is reflected or scattered to the Parachute, Sep Nuts and back shell, and causes the temperatures of these components to increase. As the back shell temperature increases, the Canister temperature increases.

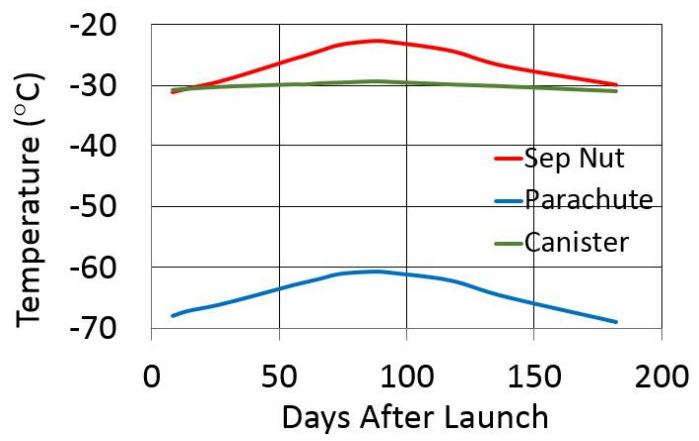

Figure 20. Flight Temperatures of SRC Sep Nut, Parachute and Canister versus Days after Launch.

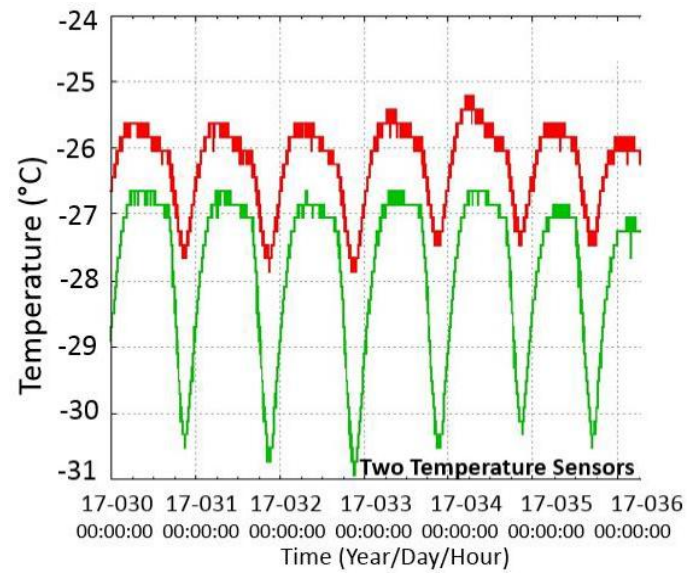

Figure 21. SRC Sep Nut Temperature from Day 030 to Day 036 in 2017.

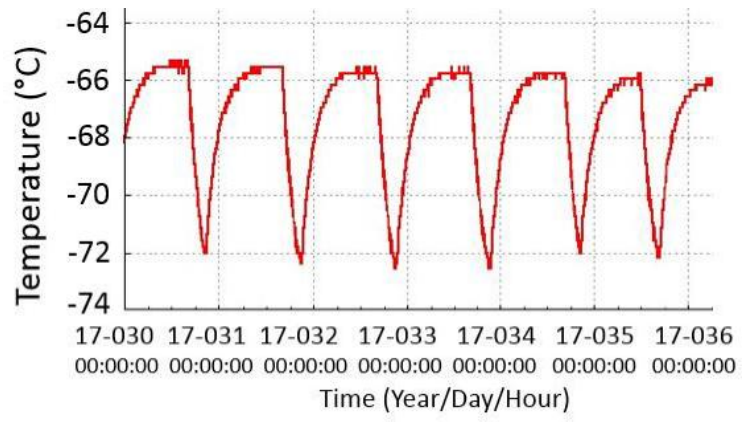

Figure 22. SRC Parachute Temperature from Day 030 to Day 036 in 2017. 


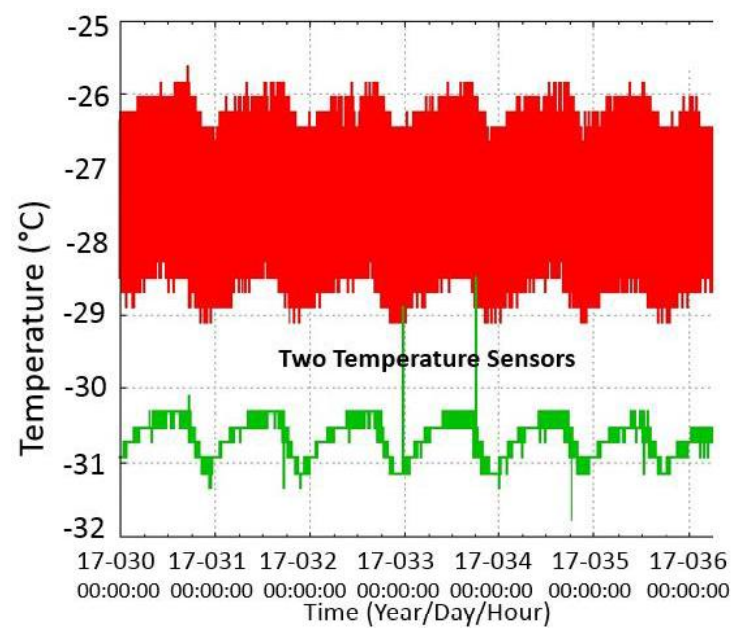

Figure 23. SRC Canister Temperature from Day 030 to Day 036 in 2017.

\subsection{Regolith X-ray Imaging Spectrometer (REXIS)}

The REXIS spectrometer has separate heater circuits on the radiation cover and frangibolt housing. They are used to maintain the frangibolt temperature above $-20^{\circ} \mathrm{C}$ in the Outbound Cruise, and until it is deployed. The heater circuits are controlled by mechanical thermostats. The thermostats on the radiation cover have a $15.9^{\circ} \mathrm{C}$ closed and $21.6^{\circ} \mathrm{C}$ open setpoint. The thermostats on the frangibolt housing have a $-19.7^{\circ} \mathrm{C}$ closed and $-11.3^{\circ} \mathrm{C}$ open set-point. From Figure 2 , the REXIS spectrometer, including the radiation cover MLI and frangibolt housing, has a view to the PolyCam and OTES sunshade MLIs. In the Outbound Cruise, the REXIS is non-operating, and has no flight temperature telemetry. The USM_3_DPC_0_CURRENT flight current telemetry is used to monitor the REXIS heater status. It is the sum of the radiation cover heater current, frangibolt housing heater current, and spacecraft Small Deep Space Transponder 1 (SDST-1) current. The power dissipation of the SDST-1 is constant at a given voltage. During the nominal Outbound Cruise spacecraft Sun-pointing attitude, the USM_3_DPC_0_CURRENT flight current telemetry is dependent on the solar distance. Figure 24 shows this trending. As the solar distance decreases, the duty cycles of the frangibolt housing and radiation cover heater circuits decrease. The frangibolt housing heater stopped drawing current 37 days after launch because its temperature was warmer than its thermostat set point. With Sun on $+X$, it resumed drawing current 125 days after launch. The relationship between solar distance and days after launch can be seen in Figure 8 . Figure 25 shows a plot of the USM_3_DPC_0_CURRENT flight current telemetry from Day 030 to Day 036 in 2017. It corresponds to Sun on $\mathrm{X}, \mathrm{Y}$ and $\mathrm{Z}$ in Figure 10. With Sun on $+\mathrm{X}$, the REXIS heater current duty cycle decreased, and often the frangibolt housing heater stopped drawing current. During the off-nominal Earth-pointing attitude, the REXIS heater current duty cycle increased, and often the frangibolt housing heater resumed drawing current. As a result, the USM_3_DPC_0_CURRENT flight current telemetry oscillated. It is evident that the REXIS spectrometer is thermally affected by solar impingement on the OCAMS PolyCam, OTES, IMU-sunshade and OLA-sunshade MLIs.

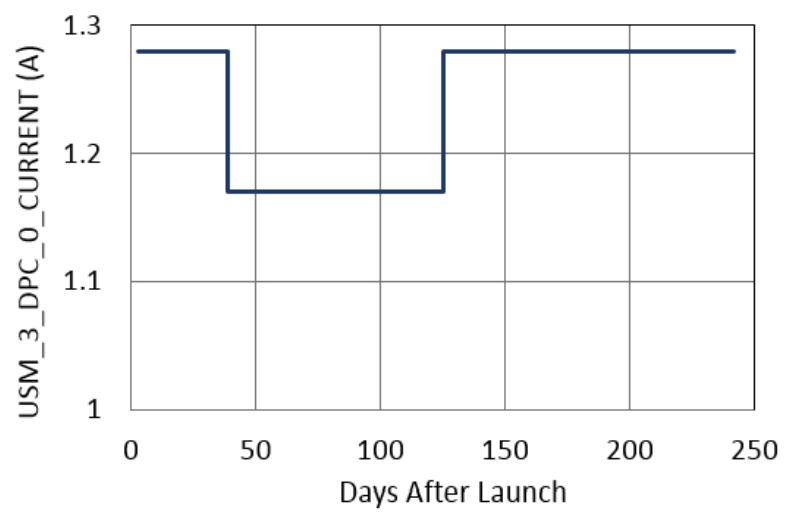

Figure 24. Maximum USM_3_DPC_0_CURRENT Flight Telemetry versus Days after Launch. 


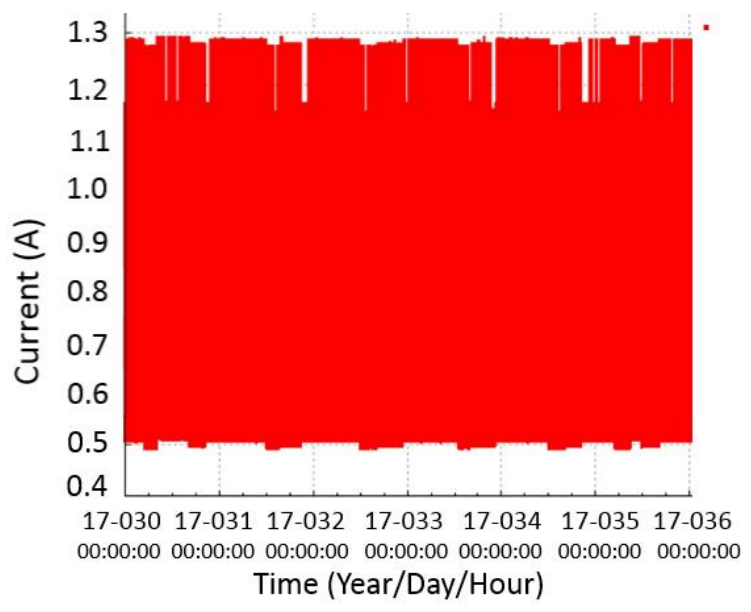

Figure 25. USM_3_DPC_0_CURRENT Flight Telemetry in Outbound Cruise from Day 030 to Day 036 in 2017.

\subsection{OSIRIS-REx Visible and Infrared Spectrometer (OVIRS)}

The OVIRS is a cryogenics temperature instrument. Its detector has a $-168^{\circ} \mathrm{C}(105 \mathrm{~K})$ maximum operating allowable flight temperature (AFT) limit, and is cooled passively by a two-stage radiator. The radiator thermal coating is Z93C55 white paint. The Optics Box is thermally isolated from a mounting bracket on the spacecraft side of the interface. The Optics Box bracket is made of carbon composite, which has a very low thermal conductivity. It is mounted to the forward deck. The detector temperature had been shown to be very sensitive to parasitic heat load during the instrument level thermal vacuum and thermal balance test. ${ }^{3}$ From Figure 2, the OVIRS Solar Calibrator and Optics Box $+Z$ side are located at the $\mathrm{X}$ edge of the forward deck. The solar calibrator protrudes above the forward deck. Both the solar calibrator and Optics Box have MLI with GBK on the exterior. Figure 26 shows the OVIRS Optics Box support bracket temperature versus days after launch. Additionally, the relationship between solar distance and days after launch can be seen in Figure 8. During the nominal Outbound Cruise spacecraft Sun-pointing attitude, the Optics Box bracket temperature increases as the solar distance decreases, and vice versa. Figure 27 shows the Optics Box bracket temperatures from Day 030 to Day 036 in 2017. It corresponds to Sun on X, Y and Z in Figure 10. When the spacecraft attitude changed from Sun-pointing to Earth-pointing, the temperatures of the Optics Box Bracket decreased, and vice versa. As a result, the temperatures of the Optics Box Bracket oscillated. Figure 28 shows the OVIRS detector cool down from Day 284 to Day 287 in 2016. During the cool down at the nominal Outbound Cruise spacecraft Sun-pointing attitude, the steady state temperature of the detector was $106 \mathrm{~K}$. It exceeded the maximum operating AFT limit by $1 \mathrm{~K}$. Additionally, it was significantly warmer than the $99 \mathrm{~K}$ predicted by the OVIRS correlated detailed thermal model. It is evident that sunlight impinging on the PolyCam, OTES, IMU-sunshade and OLA-sunshade MLIs is reflected or scattered to the OVIRS. This is illustrated in Figure 29. As a result, it increases the parasitic heat load to the detector. The OVIRS principal investigator indicated that $106 \mathrm{~K}$ is acceptable for science, despite it exceeds the maximum operating AFT limit.

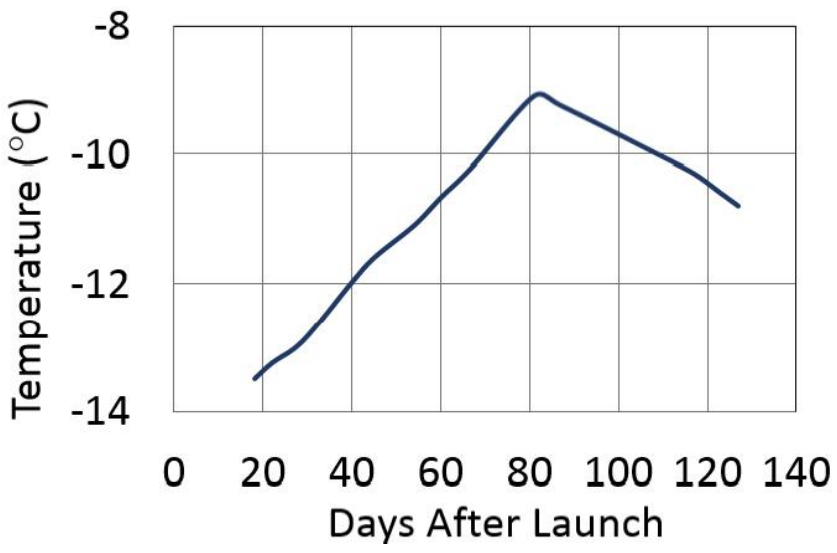

Figure 26. Flight Temperature of OVIRS Bracket in Outbound Cruise with Sun on $+X$. 


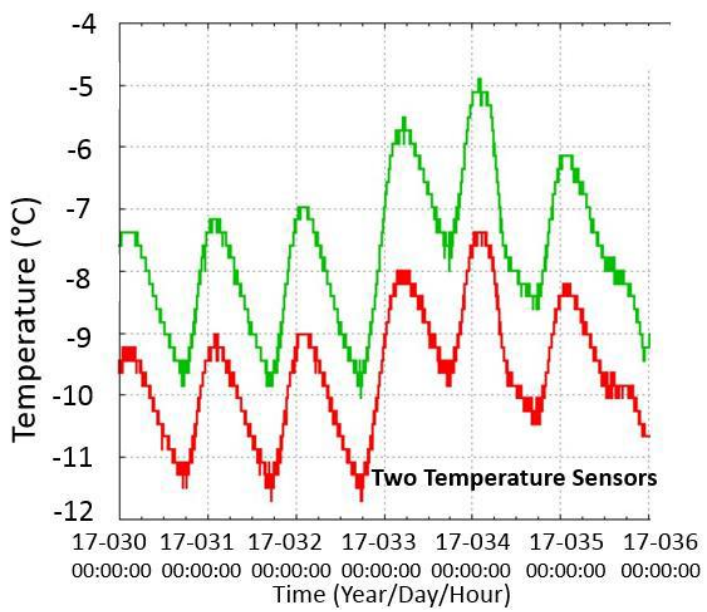

Figure 27. OVIRS Optics Box Bracket Temperature.

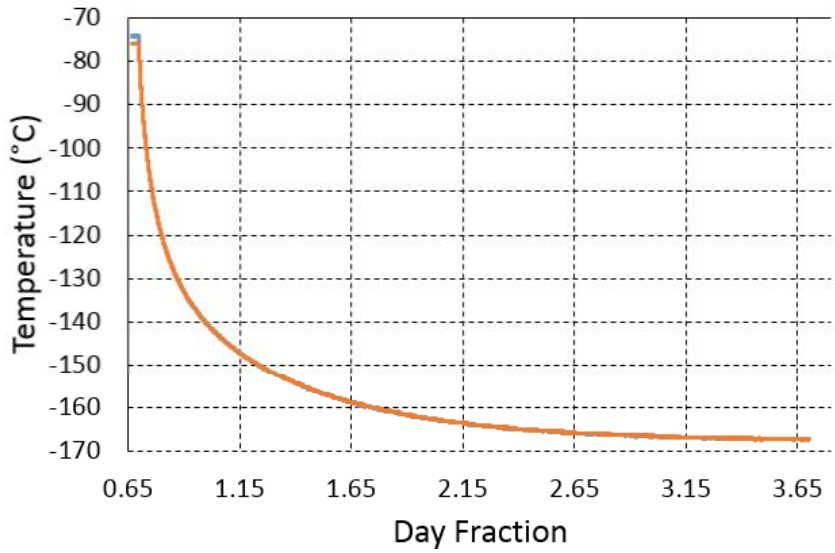

Figure 28. OVIRS Detector Cool Down in Outbound Cruise.

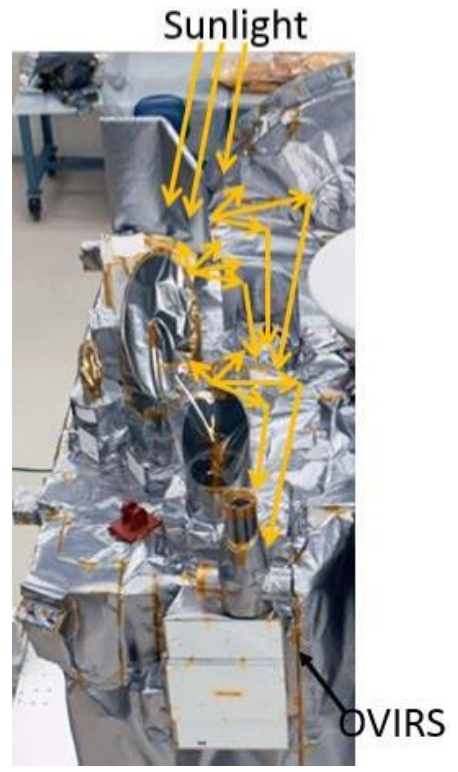

Figure 29. Reflection or Scattering of Solar Impingement to OVIRS. 


\subsection{OSIRIS-REx Thermal Emission Spectrometer (OTES)}

The OTES is located at the $+Y$ side of the forward deck (Figure 2). It is thermally isolated from its baseplate, which is on the spacecraft side of the interface, by titanium flexures. Except for the sunshade aperture, it is covered with GBK MLI blankets. It has no radiator. It radiates heat to space through the sunshade aperture to meet its thermal requirements for the detector and electronics. The survival and operating heaters are located on the instrument side and controlled by the spacecraft flight software. Figure 30 shows the OTES baseplate temperature versus days after launch. Additionally, the relationship between solar distance and days after launch can be seen in Figure 8. During the nominal Outbound Cruise Sun-pointing attitude, the OTES baseplate temperature is dependent on the solar distance. As the solar distance decreases, the OTES baseplate temperatures increase, and vice versa. Figure 31 shows the temperatures of the OTES chassis and baseplate on the spacecraft side from Day 030 to Day 036 in 2017. It corresponds to Sun on X, Y and Z in Figure 10. When the spacecraft attitude changed from Sun-pointing to Earth-pointing, the OTES baseplate temperature decreased, and vice versa. As a result, the OTES baseplate temperature oscillated. It is evident that with Sun on $+X$, sunlight impinging on the PolyCam, OTES, IMU-sunshade and OLA-sunshade MLIs is reflected or scattered to the GBK MLI over the OTES baseplate. As a result, it increases the MLI temperature, which in turns increases the OTES baseplate temperature. The OTES chassis temperature was maintained between $-15^{\circ} \mathrm{C}$ and $-11.5^{\circ} \mathrm{C}$ by its survival heaters. Changes of the spacecraft attitude from Sun-pointing to Earth-pointing, or vice versa, had a small effect on the duty cycle of the survival heaters. As the solar distance decreases, the OTES survival heater duty cycle decreases, and vice versa. It is evident that sunlight impinging on the PolyCam, OTES, IMU-sunshade and OLA-sunshade MLIs has a thermal effect on the OTES baseplate and chassis.

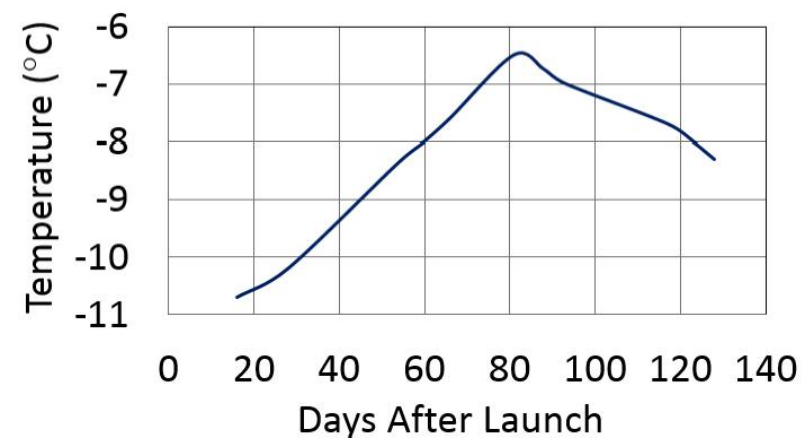

Figure 30. Flight Temperature of OTES Baseplate in Outbound Cruise with Sun on $+X$.

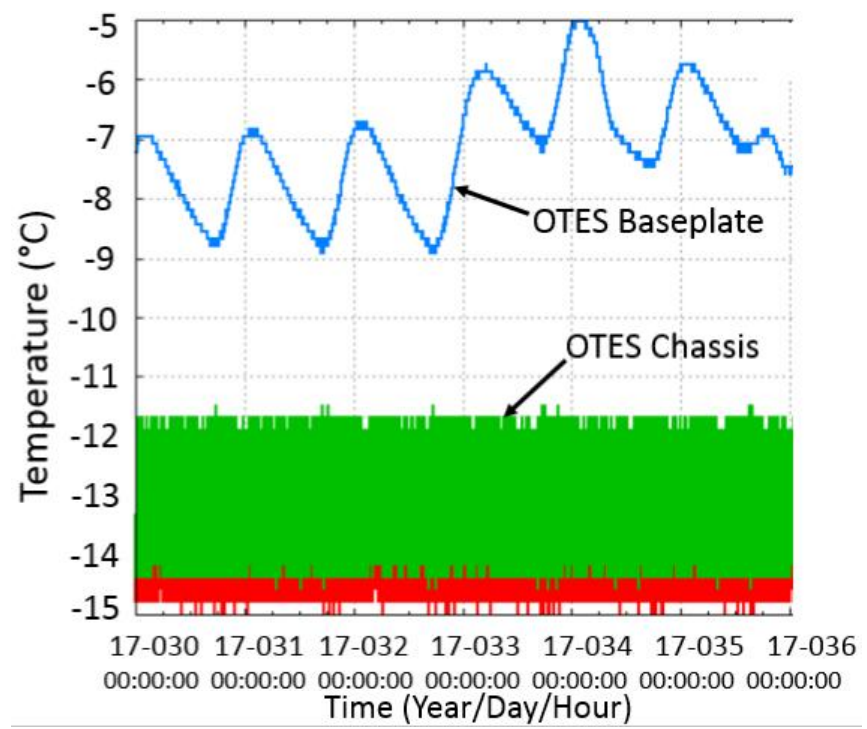

Figure 31. OTES Temperatures from Day 030 to Day 036 in 2017. 


\subsection{OSIRIS-REx Laser Altimeter (OLA)}

The OLA is located at the -Y side of the forward deck (Figure 2). It consists of an Electronics Box and a Sensor Head. Both OLA boxes are thermally coupled to their baseplates, which are integral parts of their radiators on the spacecraft side of the interface. The thermal coating is Z93C55 white paint for the Electronics Box radiator. The thermal coating is black carbon composite for the Sensor Head radiator, which is shaded from the Sun. Except for the Sensor Head window, both boxes are covered with GBK MLI blankets. The survival and operating heaters are located on the spacecraft side of the interface and controlled by the spacecraft flight software. The survival heater controller set points for the Electronics Box baseplate and Sensor Head baseplate are $-18^{\circ} \mathrm{C}$ and $-28^{\circ} \mathrm{C}$, respectively. Figure 32 shows the temperatures of the Electronics Box and Sensor Head versus days after launch. Additionally, the relationship between solar distance and days after launch can be seen in Figure 8. During the nominal Outbound Cruise Sun-pointing attitude, both the Electronics Box and Sensor Head baseplates are thermally dependent on the solar distance. After launch, the survival heaters maintained the Electronics Box baseplate between $-18^{\circ} \mathrm{C}$ and $-14^{\circ} \mathrm{C}$ until 68 days after launch. Between 68 and 128 days after launch, the survival heater power was not used because the Electronics Box baseplate temperature was warmer than $-18^{\circ} \mathrm{C}$. The survival heater resumed drawing current 268 days after launch. Since the Sensor Head baseplate temperature was warmer than the survival heater set point, survival heater power was not used. As the solar distance decreases, the Sensor Head baseplate temperatures increase, and vice versa. Figure 33 shows the temperatures of the Sensor Head baseplate from Day 030 to Day 036 in 2017. It corresponds to Sun on X, Y and Z in Figure 10. Since the spacecraft side survival heater controller set point is $-28^{\circ} \mathrm{C}$, heater power was not used. When the spacecraft attitude changed from Sun-pointing to Earth-pointing, the baseplate temperatures decreased, and vice versa. As a result, the Sensor Head baseplate temperatures oscillated. It is evident that with Sun on $+X$, sunlight impinging on the PolyCam, OTES, IMU-sunshade and OLA-sunshade MLIs is reflected or scattered to the OLA Electronics Box and Sensor Head radiators and the MLIs on the boxes.

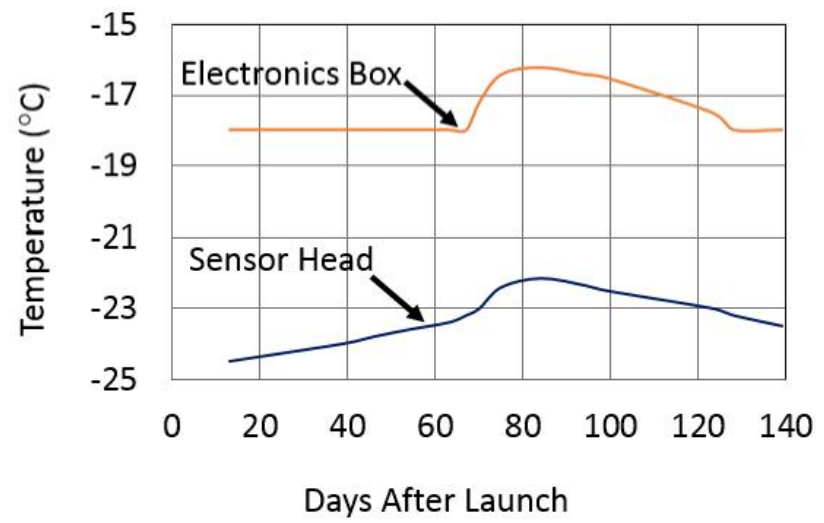

Figure 32. Flight Temperature of OLA Electronics Box and Sensor Head Baseplates in Outbound Cruise with Sun on + X.

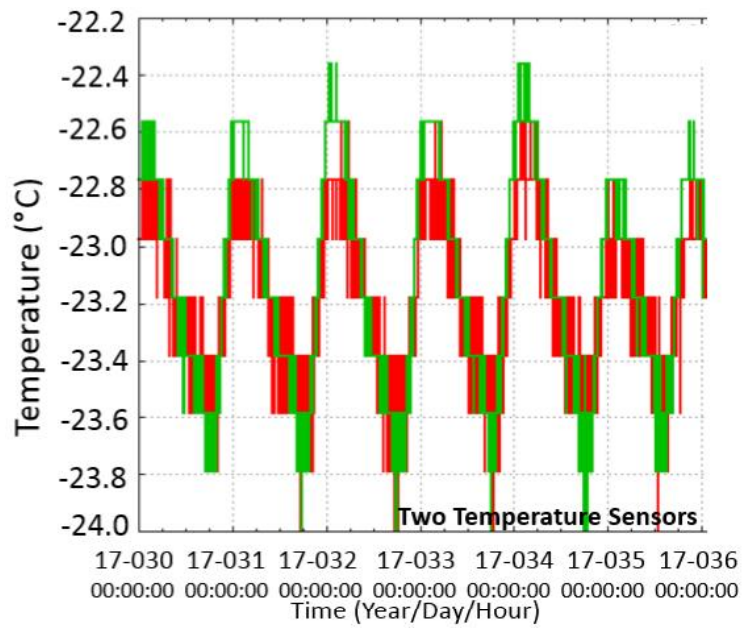

Figure 33. OLA Sensor Head Baseplate Temperatures from Day 030 to Day 036 in 2017. 


\section{THERMAL IMPLICATIONS OF SOLAR IMPINGEMENT FOR PROXIMITY OPS}

The Proximity Ops phase for flight system thermal analysis includes the Preliminary Survey, Orbital A, Detailed Survey, Orbital B and Reconnaissance (Recon) mission phases. The solar distance is in 0.89 AU to 1.387 AU range for this phase. The worst hot case solar distance is $0.89 \mathrm{AU}$ because the solar irradiance is the highest. Table 1 is a summary of the hot cases with Sun on $+X$ for thermal analysis. ${ }^{4}$

Table 1. Hot Cases with Sun on $+X$ in Proximity Ops.

\begin{tabular}{|c|c|c|c|}
\hline $\begin{array}{l}\text { Solar Off-point } \\
\text { Angle (from }+X)\end{array}$ & Mission Phase & Instruments & Description \\
\hline $0^{\circ} / 82.5^{\circ}(-\mathrm{Z})$ & Detailed Survey & Off & $+X$ Sun with 30 minute Op Navs \\
\hline $0^{\circ} / 78^{\circ}(-Z)$ & Detailed Survey & Off & $+\mathrm{X}$ Sun with 5 hour Earth-point \\
\hline $0^{\circ}$ & Detailed Survey, $90^{\circ}$ observation & On & $+\mathrm{X}$ Sun \\
\hline $0^{\circ} / 45^{\circ}(+Z)$ & Detailed Survey & Off & $+\mathrm{X}$ Sun with 30 minute Op Navs \\
\hline $0^{\circ} / 78^{\circ}(-Z)$ & Detailed Survey & Off & $+\mathrm{X}$ Sun with 5 hour Earth-point \\
\hline $0^{\circ} / 45^{\circ}(+Z)$ & Detailed Survey & Off & $+X$ Sun with 30 minute Op Navs \\
\hline $0^{\circ} / 78^{\circ}(-Z)$ & Detailed Survey & Off & $+\mathrm{X}$ Sun with 5 hour Earth-point \\
\hline $0^{\circ}$ & Orbital B, $1 \mathrm{~km}$ altitude Orbit & On & $\begin{array}{c}+\mathrm{X} \text { Sun, }+\mathrm{Z} \text { Nadir, maximum Bennu temperature } \\
\text { profile }\end{array}$ \\
\hline $0^{\circ} / 60^{\circ}$ & Orbital B, $1 \mathrm{~km}$ altitude Orbit & On & $\begin{array}{l}+\mathrm{X} \text { Sun } /+\mathrm{Z} \text { Nadir with daily Earth-point, } \\
\text { maximum Bennu temperature profile }\end{array}$ \\
\hline $0^{\circ} / 60^{\circ} / 20^{\circ}$ & Orbital B, $1 \mathrm{~km}$ altitude Orbit & On & $\begin{array}{c}+ \text { X Sun / +Z Nadir with daily Earth-point and } 6 \mathrm{x} \\
20^{\circ} \text { off-nadir Absolute Target List, maximum } \\
\text { Bennu temperature profile }\end{array}$ \\
\hline $0^{\circ}$ & Recon terminator orbit, $750 \mathrm{~m}$ altitude & Off & $+\mathrm{X}$ Sun \\
\hline Varies & $\begin{array}{l}\text { Recon, start at terminator orbit, } 0^{\circ} \\
\text { latitude pass, } 525 \mathrm{~m} \text { altitude }\end{array}$ & On & Sun angle varies \\
\hline Varies & $\begin{array}{l}\text { Recon, start at terminator orbit, } 0^{\circ} \\
\text { latitude pass, } 225 \mathrm{~m} \text { altitude }\end{array}$ & On & Sun angle varies \\
\hline
\end{tabular}

Sunlight impinges on the PolyCam, OTES and IMU-sunshade and OLA-sunshade MLIs in the Outbound Cruise. It is reflected or scattered to the other components on the forward deck. It could contribute to degradation of the optical properties of thermal coatings, including GBK and Z93C55 white paint. The end-of-life (EOL) optical properties for thermal analysis are deemed conservative to cover this degradation. The thermal implications of the above solar impingement to the components on the forward deck in the Proximity Ops are discussed below.

\subsection{REXIS}

For the REXIS spectrometer, with the radiation cover deployed, the coded mask and mask frame in Proximity Ops are expected to receive both infrared heat radiation and sunlight reflected or scattered from the PolyCam and OTES sunshade MLIs. The radiation cover was closed for entire flight system thermal vacuum (TVAC) test, and the above Proximity Ops configuration was not simulated. Additionally, the flight system TVAC had only 1.1 Sun (0.97 AU) maximum due to solar simulator problems. The correlated flight system thermal model ${ }^{4}$ predicts the CCD maximum operating temperature in the Proximity Ops to be $-73^{\circ} \mathrm{C}$, which is $18^{\circ} \mathrm{C}$ below the maximum operating AFT limit of $-55^{\circ} \mathrm{C}$. Due to infrared heat radiation and sunlight reflected or scattered to the spectrometer from the PolyCam and OTES sunshade MLIs, the CCD temperature margin could be reduced, but its maximum operating temperature is expected to be well below the maximum operating AFT limit. 


\subsection{OVIRS}

Sunlight impinging on the PolyCam, OTES, IMU-sunshade and OLA-sunshade MLIs is reflected or scattered to the OVIRS Solar Calibrator and Optics Box $+Z$ side MLIs. During the OVIRS detector cool down from Day 284 to Day 287 in 2016, the solar distance was $0.897 \mathrm{AU}$ when the cool down began and $0.887 \mathrm{AU}$ when the cool down ended. The worst hot case solar distance in the Proximity Ops is $0.89 \mathrm{AU}$, which is basically the same as that during the OVIRS cool down in the Outboard Cruise. The optical properties of the GBK MLI could have degradation from launch to the Proximity Ops. Therefore, the effect of the above solar impingement on the detector temperature in the Proximity Ops is not expected to be significantly larger than that of the Outboard Cruise. Additionally, the Bennu asteroid flux is expected to add warming to the OVIRS detector, especially in the Reconnaissance phase. As a result, the OVIRS detector temperature in the Reconnaissance phase of the Proximity Ops is expected to be approximately $4 \mathrm{~K}$ warmer than the 106K steady state temperature achieved in the Outbound Cruise.

\subsection{OCAMS}

Sunlight impinging on the PolyCam, OTES and IMU-sunshade MLIs is reflected or scattered to the forward deck MLIs and the MapCam, PolyCam and SamCam chassis MLIs. Assuming the optical properties of the GBK of the MLIs at the EOL, it will increase the temperatures of the forward deck, MapCam, PolyCam and SamCam baseplates, and MapCam, PolyCam and SamCam chassis. The baseplates of the cameras are on the spacecraft side of the forward deck, and are thermally isolated from the camera chassis by titanium flexures. The MapCam and PolyCam radiators are located on the $-\mathrm{X} /+\mathrm{Y}$ side of the cameras (Figure 6). Reflection or scattering of sunlight impinging on the OTES MLIs to these radiators is not negligible. Additionally, these radiators have a small view factor to the OTES MLIs. The SamCam radiator has a view factor, either directly or through GBK's specular reflections, with the PolyCam and OTES MLIs. The flight system TVAC had only 1.1 Sun $(0.97 \mathrm{AU})$ maximum due to solar simulator problems. The solar irradiance in the Proximity Ops hot case is higher (1.26 Sun at $0.89 \mathrm{AU}$ ) and the optical properties of thermal coatings could have degradation. Therefore, the temperatures of the MapCam, PolyCam and SamCam CCDs and PolyCam Optics are expected to be warmer than those of the flight system TVAC. However, the correlated flight system thermal model predictions have adequate margins for these components. The operating temperature predictions on the MapCam, PolyCam and SamCam CCDs have at least $17^{\circ} \mathrm{C}, 21^{\circ} \mathrm{C}$ and $10^{\circ} \mathrm{C}$ margins for the PolyCam, MapCam and SamCam, respectively. The operating temperature predictions on the MapCam, PolyCam and SamCam chassis have at least $24^{\circ} \mathrm{C}$, $45^{\circ} \mathrm{C}$ and $29^{\circ} \mathrm{C}$ margins for the PolyCam, MapCam and SamCam, respectively. Due to infrared heat radiation and sunlight reflected or scattered to the OCAMS cameras from the PolyCam, OTES sunshade and OLA-sunshade MLIs, the OCAMS temperature margins could be reduced, but the maximum operating temperatures are expected to be below the maximum operating AFT limit.

\subsection{TAGCAMS}

Sunlight impinging on the PolyCam, OTES, IMU-sunshade and OLA-sunshade MLIs is reflected or scattered to the StowCam. The flight system TVAC had only 1.1 Sun (0.97 AU) maximum due to solar simulator problems. The solar irradiance in the Proximity Ops hot case is higher (1.26 Sun at $0.89 \mathrm{AU})$ and the optical properties of thermal coatings could have degradation. Assuming the optical properties of the GBK of the MLIs and Z93C55 white paint radiator at the EOL, it will increase the temperatures of the StowCam. Additionally, the StowCam has a view factor to the PolyCam and OTES MLIs. Therefore, the temperature of the StowCam is expected to be warmer than those of the flight system TVAC. The correlated flight system thermal model predictions have adequate margins for these components. The StowCam is non-operating in the Proximity Ops phase. The non-operating temperature of the StowCam has at least a $27^{\circ} \mathrm{C}$. The NavCam is operating in the Survey Cases of the Proximity Ops phase. The operating temperature of the NavCam has at least a $5^{\circ} \mathrm{C}$ margin. There are adequate margins to cover the infrared heat radiation and sunlight reflected or scattered to the StowCam and NavCam by the PolyCam, OTES, IMU-sunshade and OLA-sunshade MLIs.

\subsection{OTES}

The OTES is thermally isolated from its baseplate by titanium flexures. It has no radiator. It radiates heat to space through the sunshade aperture to cool the detector and electronics. Except for the aperture, the OTES is covered with MLIs. Despite sunlight impinging the PolyCam, OTES, IMU-sunshade and OLA-sunshade MLIs is reflected or scattered to the OTES MLI and baseplate MLI, the thermal effect on OTES is not significant. The correlated flight system thermal model predictions have adequate margins. The OTES worst hot case temperature prediction has at least a $27^{\circ} \mathrm{C}$ margin in the Proximity Ops. It is adequate to cover the sunlight reflected or scattered to the OTES MLI and its baseplate MLI by the PolyCam, OTES, IMU-sunshade and OLA-sunshade MLIs at the EOL optical properties. 


\subsection{OLA}

The thermal coating is Z93C55 white paint for the Electronics Box radiator. The thermal coating is black carbon composite for Sensor Head radiator, which is shaded from the Sun. The flight system TVAC had only 1.1 Sun (0.97 AU) maximum due to solar simulator problems. Additionally, the thermal coating for the Sensor Head radiator was Z93C55 white paint in the flight system TVAC. It was removed after the TVAC due to its delamination problem. The correlated flight system thermal model predictions have adequate margins. The Electronics Box and Sensor Head worst hot case operating temperatures have at least a $15^{\circ} \mathrm{C}$ and a $5^{\circ} \mathrm{C}$ margin, respectively, in the Proximity Ops. Sunlight reflected or scattered by the PolyCam, OTES, IMU-Sunshade and OLA-sunshade MLIs at the EOL optical properties are not expected to cause AFT limit violations for OLA Electronics Box or Sensor Head.

\subsection{SRC}

The SRC components are non-operating in the Proximity Ops phase. The flight system TVAC had only 1.1 Sun $(0.97$ AU) maximum due to solar simulator problems. The correlated flight system thermal model predictions have adequate margins in the Proximity Ops. The worst hot case non-operating temperatures for the Parachute, Sep/Spin mechanism interface, Sep Nuts and Canister have a margin of least $29^{\circ} \mathrm{C}, 41^{\circ} \mathrm{C}, 83^{\circ} \mathrm{C}$ and $76^{\circ} \mathrm{C}$, respectively. The forward deck maximum temperature at the SRC Sep/Spin interface has a $20^{\circ} \mathrm{C}$ margin. Infrared heat radiation and sunlight reflected or scattered by the PolyCam, OTES, IMU-sunshade and OLA-sunshade MLIs to the SRC components at the EOL optical properties are not expected to cause AFT limit violations for the SRC.

\section{THERMAL IMPLICATIONS OF SOLAR IMPINGEMENT FOR TAG}

The TAG Phase covers the TAG Rehearsal phase through the successful TAG sample collection, sample mass measurement and sample stow. ${ }^{5}$ During the TAG Phase, the solar distance is in the 0.897 AU to 1.387 AU range. The Safe Mode attitude for the TAG is same as that for the Outbound Cruise nominal attitude with $+\mathrm{X}$ points at Sun. For thermal analysis, the TAG begins in a $1 \mathrm{~km}$ safe home orbit. The spacecraft attitude is $+X$ Sun-pointing, with a 2 hour off-nominal attitude, which puts the Sun on the $-Z$ deck $\left(50^{\circ}\right.$ with $\left.-Z\right)$. The next part of the simulation starts after the departure burn with the $+X$ axis towards the Sun, continues through the Natural Feature Tracking attitude with Sun offnominal (approximately $17.7^{\circ}$ from $+\mathrm{X}$ ), and through the Look-Ahead attitude with sun approximately $55^{\circ}$ from $+\mathrm{X}$. After slewing to the final TAG attitude 36 minutes before the TAG, this part of the simulation ends at the Checkpoint maneuver. The TAG attitude has Sun $25^{\circ}$ from $+X$ (i.e., $65^{\circ}$ with $-Z$ axis). The OLA and REXIS are non-operating in the TAG phase. The OCAMS (MapCam, PolyCam and SamCam), OVIRS and NavCam are operating during the calibration subphase. Only the NavCam is operating during the Safe Home Orbit (OpNav) subphase. The MapCam, SamCam, OTES, OVIRS and NavCam are operating during the TAG subphase. The OTES and OVIRS collect science data beyond the baseline mission, and are best effort only. The SamCam and StowCam are operating during the Checkout/Stow subphase. The optical properties of thermal coatings, including GBK and Z93C55 white paint, may continue to degrade over time. Reflection or scattering of the solar impingement on the PolyCam, OTES, IMU-sunshade and OLA-sunshade

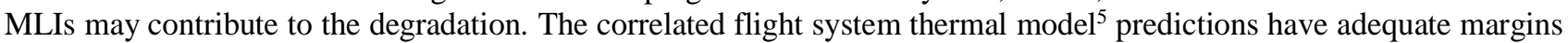
in the TAG phase, except for the OVIRS detector. The margins for the worst hot operating case MapCam CCD, PolyCam CCD, SamCam CCD, NavCam, StowCam and OTES temperatures are $35^{\circ} \mathrm{C}, 45^{\circ} \mathrm{C}, 38^{\circ} \mathrm{C}, 33^{\circ} \mathrm{C}, 52^{\circ} \mathrm{C}$ and $19^{\circ} \mathrm{C}$, respectively. There are adequate margins to cover the infrared heat radiation, and sunlight reflected or scattered by the PolyCam, OTES, IMU-sunshade and OLA-sunshade MLIs to the above components and forward deck MLI, all at the EOL optical properties. The worst hot operating case OVIRS detector temperature prediction is $80 \mathrm{~K}$ warmer than its maximum operating AFT limit. It is due to the TAG attitude and asteroid Bennu.

\section{THERMAL IMPLICATIONS OF SOLAR IMPINGEMENT FOR RETURN CRUISE}

The Safe Mode attitude for the Return Cruise is the same as that for the Outbound Cruise (i.e., nominal Sun-pointing attitude (Sun on $+\mathrm{X})$ ). Additionally, the solar distance for the Outbound Cruise is the same as that for the Outbound Cruise (i.e., from $0.773 \mathrm{AU}$ to $1.387 \mathrm{AU}$ ). In the Return Cruise, all the instruments are powered off and the survival heaters for all the instruments are disabled. The AFT limits will no longer apply to the instruments in the Return Cruise. The survival heaters for the TAGCAMS are enabled. The optical properties of thermal coatings, including GBK and Z93C55 white paint, may continue to degrade over time. Reflection or scattering of the sunlight impinging on the PolyCam, OTES, IMU-sunshade and OLA-sunshade MLIs may contribute to the degradation. At the end of the Outbound Cruise, the SRC will be released for Earth re-entry. The SRC avionics and battery are in the operating mode 
during the SRC Release. The EOL optical properties affect the SRC avionics and battery temperatures. The first SRC Optical Properties Characterization was performed from Day 053 to Day 054 at $1 \mathrm{AU}$. The spacecraft was slewed to a thermally equivalent SRC Release attitude and remained there until the battery temperature reached the pre-defined threshold. The second SRC Optical Properties Characterization will be repeated in the Return Cruise. It will provide a good characterization of any changes (i.e., a regolith deposit or other degradation) in optical properties that might have occurred during the TAG, or during a long time in space. If the SRC battery temperature is much warmer during the second characterization, it will be necessary to make some changes to the SRC Release timeline so that the SRC battery will not get too warm too soon.

\section{CONCLUSION}

The OSIRIS-REx Outbound Cruise flight temperature telemetry and USM_3_DPC_0_CURRENT flight current telemetry data have been analyzed. It is evident that at the nominal Outbound Cruise spacecraft Sun-pointing attitude (i.e., Sun on $+\mathrm{X}$ ), sunlight impinging on the PolyCam, OTES, IMU-sunshade and OLA-sunshade MLIs is reflected or scattered to the forward deck and components on the forward deck. It illuminates the forward deck. The StowCam image of Day 2652016 also provided an evidence. The reflected or scattered sunlight cause warming to the forward deck and components on its $+Z$ side. It may also contribute to degradation of thermal coatings over the mission life. It is a factor that the OVIRS detector operating temperature exceeds the $105 \mathrm{~K}$ maximum AFT limit. The OVIRS Principal Investigator indicated that it is not optimum but acceptable for science. With exception of the OVIRS detector, the correlated flight system thermal model predictions for the components on the forward deck have adequate margins in the Proximity Ops, TAG and Return Cruise phases. The margins are expected to cover the warming caused by the solar impingement and the contribution to degradation of thermal coatings. The solar impingement is not expected to be a thermal risk to the OSIRIS-REx mission. The second SRC Optical Properties characterization will be repeated in the Return Cruise to provide a good characterization of any changes in optical properties that might have occurred during the TAG, or during several years in space. If the SRC battery runs much warmer than that of the first characterization in the Outbound Cruise, it will be necessary to make some changes to the SRC Release timeline to assure the SRC battery temperature are within limits. If GBK, instead of microporous black PTFE, were used on the PolyCam sunshade MLI, much more sunlight would have been reflected or scattered to the forward deck and components on its $+Z$ side.

Microporous black PTFE should be considered to mitigate the optical and thermal issues of sunlight reflected/scattered by MLI blankets in future missions.

\section{REFERENCES}

[1] Choi, M. K., "Using microporous polytetrafluoroethylene thin sheets as a flexible solar diffuser to minimize sunlight glint to cameras in space", Proc. SPIE 9981-13, (2016).

[2] Rizk, B., "OSIRIS-REx Post-Launch Assessment Review: \#9 - OCAMS L+14 Check-out," Oct. 25, 2016.

[3] Caldwell, E. and Chang, W., "OSIRIS-REx Project OSIRIS-REx Visible and near-IR Spectrometer (OVIRS) Thermal Vacuum Test Report TV\#1 and TV\#2", PLA-OVIRS-RPT-0059 (2015), NASA GSFC.

[4] Church, M., et al., "Proximity Ops Thermal Analysis," NFP3-AN-13-1236, Lockheed Martin June 20, 2016.

[5] Stoll, M., et al., "TAG Thermal Analysis," NFP3-AN-14-1237, Lockheed Martin June 27, 2016. 